\title{
Regions of attainable particle sizes in continuous and batch crystallization processes
}

\author{
Thomas Vetter ${ }^{\mathrm{a}, \mathrm{b}}$, Christopher L. Burcham ${ }^{\mathrm{b}}$, Michael F. Doherty ${ }^{\mathrm{a}, *}$ \\ ${ }^{a}$ Department of Chemical Engineering, University of California, Santa Barbara, California \\ 93106 \\ ${ }^{b}$ Eli Lilly $\& 3$ Company, Indianapolis, Indiana 46285
}

\begin{abstract}
Process alternatives for continuous crystallization, i.e., cascades of mixed suspension, mixed product removal crystallizers (MSMPRCs) and plug flow crystallizers (PFCs), as well as batch crystallizers are discussed and modeled using population balance equations. The attainable region approach that has previously been used in the design of chemical reactor networks and separation systems is applied to the above-mentioned alternatives for crystallization processes in order to identify attainable regions in a diagram of mean product particle size vs. total process residence time. It is demonstrated that the boundaries of these attainable regions can be found numerically by solving appropriate optimization problems and that the region enclosed by these boundaries is fully accessible. Knowing the attainable region of particle sizes, it is possible to generate feasible process alternatives that allow specific particle sizes to be obtained in a given process configuration. The attainable regions presented in this article are useful to determine whether a desired mean particle size can be achieved in a specific crystallizer type. The concept of the attainable region is illustrated on three case studies: the cooling crystallization of paracetamol grown from ethanol, the anti-solvent crystallization of L-asparagine monohydrate from water using isopropanol as the anti-solvent and the combined cooling/anti-solvent crystallization of aspirin from ethanol using water as the anti-solvent.
\end{abstract}

Keywords: attainable region, process design, particle size distribution, Continuous crystallization, batch crystallization

\footnotetext{
* Corresponding author

Email address: mfd@engineering.ucsb.edu (Michael F. Doherty)
} 


\section{Introduction}

Crystallization is widely used in the separation and purification of commodities, fine chemicals and active pharmaceutical ingredients (APIs). While the purity of the produced crystals is the primary concern in all of these applications, there are secondary properties, such as the crystal form and the particle size (and shape) distribution of the product crystals, that need to be considered as well. While these secondary properties merely affect further processing steps in the case of chemical intermediates (e.g., adipic acid), they are crucial in many other cases, among them the production of pigments (Brazeau et al., 2011; Liu et al., 2010), where the color, its intensity and brilliance depend on the particle size distribution and in the pharmaceutical industry, where the dissolution properties, the bioavailability and and even the biocompatibility (Cavalcante et al., 2009) of an API are influenced by its particle size distribution.

In the pharmaceutical sector the vast majority of crystallization processes have been carried out for decades as batch processes and that processing method remains prevalent today (Chen et al., 2011). It is recognized that this type of operation suffers from product variability from batch-to-batch and potentially high manufacturing costs (Lawton et al., 2009; Randolph and Larson, 1988). The operation of batch crystallization processes is however quite complex and advanced control strategies are required in order to consistently fulfill product specifications, e.g., a desired particle size distribution (Nagy and Braatz, 2012). In contrast, continuous processes operate at steady state for which a plethora of well-established control strategies is available.

While continuous processing is a proven technique in many large scale industries for overcoming batch-to-batch variabilities and to ensure low-cost production, the pharmaceutical industry has been reluctant to embrace continuous manufacturing for two main reasons: first, the pharmaceutical industry is subject to a unique set of (regulatory) challenges; second, the low production volumes and the existing batch production capabilities rarely have justified building a dedicated continuous manufacturing plant for the production of a specific API. However, as global competition increases there is now an increased focus on reducing manufacturing costs while maintaining the high product quality that fulfills the regulatory demands.

The design methodology for batch crystallization processes has been investigated extensively and is now well understood for various combinations of cooling, anti-solvent and reactive crystallization (Genck, 2003; Larsen et al., 2006; Lindenberg et al., 2009) and for different optimization objectives (Nagy et al., 2008; Ward et al., 2006; Nagy and Braatz, 2012; Rawlings et al., 1993) (note that the list of references given is by no means exhaustive). However, there are yet relatively few studies targeted on the design and optimization of continuous crystallization processes that keep the specific challenges posed to the pharmaceutical industry in mind, e.g., Alvarez and Myerson (2010) presented a plug flow crystallizer (PFC) with incorporated static mixing elements that was used in the anti-solvent crystallization process of the API ketoconazole, and Eder et al. (2010) presented a continuously seeded PFC that was used in 
the cooling crystallization of aspirin from ethanol. In PFCs one of the main complications is the need to keep the crystals suspended; a feat that is typically achieved by running the PFC at high flow rates resulting in turbulent flow behavior within the pipe. Hence, using a PFC at the (low) production rates prevalent in the pharmaceutical industry is often impractical. However, Lawton et al. (2009) showed that issues with suspension can sometimes be cleverly circumvented using a continuous oscillatory baffled crystallizer resulting in a process that performs similar to an ideal PFC without the limitation of high flow rates. As an alternative, continuous crystallization processes can be operated in mixed suspension, mixed product removal crystallizers (MSMPRCs). Due to their simpler operation, they have been used in a pharmaceutical context in various configurations (single stage, multi stage, with and without recycling operations, etc.) (Wong et al., 2012; Zhang et al., 2012; Alvarez et al., 2011; Quon et al., 2012) and in combination with additional product classification equipment or fines destruction loops (Griffin et al., 2010; Mersmann, 2001).

While these studies clearly show the applicability of continuous crystallization processes in the pharmaceutical industry and can serve as case studies, a process design methodology is still largely missing. In this article, we make a contribution to such a methodology by reporting the influence of the number of stages, as well as the temperature, anti-solvent fraction and residence time in each crystallizer on the particle size distribution of the product. Specifically, we demonstrate that for any number of MSMPRCs and a constant production rate there exists a clearly defined attainable region in a diagram of mean particle size of the product crystals vs. total residence time in the MSMPRC cascade. Using extensive simulations, it will be shown that this attainable region can be entirely traversed by altering the temperature, solvent composition and residence time in each MSMPRC. Moreover, such attainable regions can also be determined for PFCs and semi-batch crystallizers by slightly adjusting the methodology used for MSMPRC cascades.

The remainder of this paper is structured as follows: In Section 2 the flowsheets and processing variants considered will be presented. Section 3 summarizes the population balance equation models used to describe the evolution of the crystal size distribution in the different crystallizers. In Section 4 the concept of the attainable region is introduced and adapted to crystallization processes. The methodology to construct these attainable regions is also explained. Finally, in Section 5 the attainable regions for different crystallizer setups (MSMPRC cascade, PFC and semi-batch) are presented for three different case studies: the cooling crystallization of paracetamol from ethanol, the anti-solvent crystallization of L-asparagine monohydrate from water using isopropanol as the anti-solvent and the combined cooling/anti-solvent crystallization of aspirin from ethanol using water as the anti-solvent. The results section of this paper is concluded by presenting the effect of additional operational constraints on the attainable regions. 


\section{Flowsheets for continuous and batch crystallization processes}

In the pharmaceutical industry crystallization processes are usually carried out in a (semi-)batch device which is seeded and operated at low supersaturation, so that the formation of additional nuclei is avoided and the crystallization process is "growth controlled". Such an operating policy ideally yields a unimodal size distribution in which the final size of the crystals can be conveniently tuned by choosing seed mass and size (Chung et al., 1999; Ward et al., 2006, 2011) The mean particle size of the product follows the expression $\bar{L}_{f}=\left(m_{f} / m_{s}\right)^{1 / 3} \bar{L}_{s}$ where $m_{f}$ is the mass of isolated crystalline product, $m_{s}$ is the seed mass and $\bar{L}_{s}$ is the mean size of the seed crystals. ${ }^{1}$ Another distinct advantage of batch operating policies is the tight control over the crystal form (i.e., which polymorph, solvate, etc. is produced), which can be ensured by seeding the process with the desired crystal form. In a continuous process, using a classical seed procedure is impractical (and even unnecessary), so that an operating policy must include accurate knowledge of nucleation at the steady state conditions of the continuous process. In this work, we assume that nucleation behaves in a deterministic manner on the scale of the whole crystallizer, i.e., nuclei are formed at a constant rate by primary and/or secondary nucleation when the crystallizer is at steady state conditions. This assumption breaks down only for very low nucleation rates or low process volumes where the stochastic nature of nucleation becomes apparent (Kadam et al., 2011, 2012). In continuous processing, compounds that exhibit negligible nucleation rates at reasonable supersaturation levels present a challenge, as these low nucleation rates cannot sustain an appropriate number of crystals in the crystallizer. Hence, alternative ways of "nuclei generation" must be devised, such as the formation of nuclei at high supersaturations in impinging jet mixers (Woo et al., 2011) or the use of a wet mill (Kougoulos et al., 2011) to break down larger particles. The particles produced with the impinging jet mixers could then be added to the MSMPRC or PFC, while a wet mill could be used in a recycle loop where part of the product particles are fed back through the mill to undergo breakage before entering an MSMPRC cascade or a PFC. While these two techniques work in some cases, they also have their pitfalls, i.e., the formation of nuclei at high supersaturations in impinging jet mixers can lead to the nucleation of metastable crystal forms while wet milling can sometimes accelerate solvent mediated phase transformations. These advanced schemes for nuclei generation along with many other process variants, such as recycle operations, product classifiers, fines dissolution loops, etc., will not be considered in this work. However, the methodology presented in the following sections could be extended to these process schemes.

In this work, only the basic process alternatives are considered, i.e., one pass cascades of MSMPRCs, one pass PFCs and semi-batch crystallizers. Note also that for semi-batch crystallizers only unseeded variants are considered to

\footnotetext{
${ }^{1}$ Note that in this equation we have assumed that the crystal shape does not change and that all crystallized mass is deposited on the seed crystals.
} 
put them on an equal footing with the (unseeded) continuous alternatives. The flowsheets for these process variants are shown in Figure 1. One can see that all flowsheets allow cooling and the addition of anti-solvent through the streams labeled $F_{i}, f(z)$ and $F(t)$, respectively, in order to induce crystallization. Note that $F_{i}$ and $F(t)$ signify volumetric flow rates, while $f(z)$ is a volumetric flow rate per unit length, i.e., it is a differential side stream that allows a continuous addition of anti-solvent along the length of the PFC (see also Section 3). At steady state (in the case of the MSMPRC and PFC) the state of the crystallizer is mainly characterized by the particle size distribution it contains $(n)$, the solute concentration in the liquid phase $(c)$, the anti-solvent volume fraction in the liquid phase $(a)$, the temperature $(T)$ and, in the case of the semi-batch and MSMPRC, the volume of suspension $(V)$. Note that the driving force of a crystallization process is the difference in chemical potential between solid and liquid phase, which is often expressed through the supersaturation $S=c / c_{\star}$ where $c$ is the solute concentration and $c_{\star}(T, a)$ is the equilibrium solubility. The feed and outflowing streams to the MSMPRC and PFC are characterized by their volumetric flow rates $(Q)$, their temperature and their solute and antisolvent concentrations. 


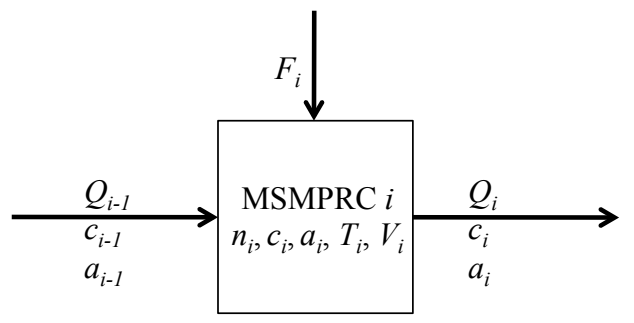

(a)

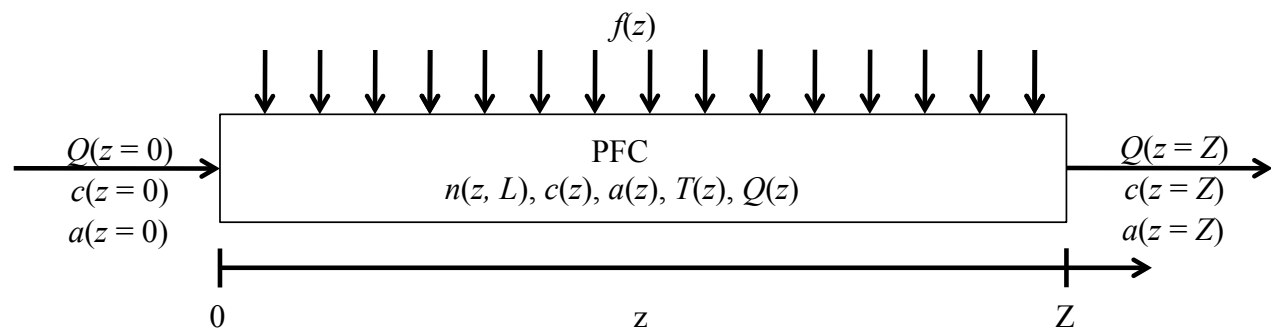

(b)

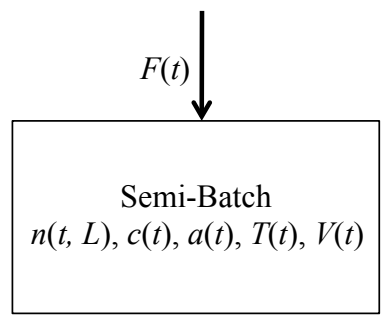

(c)

Figure 1: Flowsheet alternatives considered in this work: a) mixed suspension mixed product removal crystallizer (MSMPRC) that is part of a cascade, (b) plug flow crystallizer (PFC) with anti-solvent addition through a differential side stream, (c) semi-batch crystallizer, 


\section{Process modeling}

To model the crystallization process in the flowsheets described in the previous section, population balance equation (PBE) models are used (Ramkrishna, 2000; Rawlings et al., 1993; Randolph and Larson, 1988). For an MSMPRC cascade consisting of $m$ stages (as shown in Figure 1a) a general form of the PBE can be written as:

$$
\frac{\partial n_{i}}{\partial t}=-\frac{\partial\left(G n_{i}\right)}{\partial L}+\frac{Q_{i-1} n_{i-1}-Q_{i} n_{i}}{V_{i}}+B-D \quad \text { for } \quad i=1, \ldots, m
$$

where $n_{i}(t, L)$ is the number density distribution in the $i^{\text {th }}$ MSMPRC, such that $n_{i} \mathrm{~d} L$ represents the number of particles per unit volume with characteristic sizes between $L$ and $L+\mathrm{d} L, G$ is the overall growth rate of the crystals, $Q_{i}$ is the volumetric flow rate of the $i^{\text {th }}$ stream, $V_{i}$ is the volume of suspension and $B$ and $D$ are birth and death rate terms that account for nucleation, agglomeration and breakage. To arrive at this equation, it was further assumed that every MSMPRC

- is well-mixed,

- that the sums of the volumetric flow rates entering and leaving the MSMPRC are equal (i.e., the mixture of solute, solvent and anti-solvent is an ideal solution and that crystallization does not change the volume of suspension),

- and that all properties of the outflowing stream are identical to the conditions inside the MSMPRC.

In the context of this work, only the steady state behavior of the crystallization process is of interest and agglomeration and breakage are assumed to be negligible. Eq. (1) can then be simplified to obtain:

$$
0=-\frac{\partial\left(G n_{i}\right)}{\partial L}+\frac{Q_{i-1} n_{i-1}-Q_{i} n_{i}}{V_{i}} \quad \text { for } \quad i=1, \ldots, m
$$

with the initial, boundary and regularity conditions given as

$$
\begin{aligned}
& n_{i}(L=0) \\
& n_{i}(L=\infty) \quad=\quad \bar{G} \\
&
\end{aligned}
$$

where nucleation has been expressed in the boundary condition instead of a source term. Note that $G$ and $J$ are typically nonlinear functions of the solute concentration and temperature and that $J$ can also depend on the particle size distribution itself (e.g., in the case of secondary nucleation). It is wellknown that depending on the nucleation mechanisms considered an MSMPRC can exhibit multiple steady states (Sherwin et al., 1967; Yu and Douglas, 1975;

Lakatos, 1996; Lakatos et al., 2007). However, in this work case studies will 
be presented that admit unique steady states, such that the initial state of the crystallizer can be disregarded as it will not affect the steady state solution.

For the PFC shown in Figure 1b the steady state PBE, considering equivalent simplifying assumptions as in the MSMPRC, can be written as:

$$
0=-\frac{\partial(G n)}{\partial L}-\frac{1}{A} \frac{\partial(Q n)}{\partial z}
$$

where $n(z, L)$ is the number density distribution, $A$ is the cross sectional area of the pipe, $z$ is the length coordinate along the PFC and $Q(z)$ is the volumetric flow rate in the PFC. In order to arrive at this equation it was assumed that the anti-solvent is added using a differential side stream $(f(z)$ in Figure 1b), such that $Q n$ is continuously differentiable with respect to $z$ over the whole length of the PFC. Eq. (4) can then be rewritten as:

$$
\frac{Q}{A} \frac{\partial n}{\partial z}=-\frac{\partial(G n)}{\partial L}-\frac{f n}{A}
$$

where we have used $f=\partial Q / \partial z$. In practice, adding the anti-solvent continuously along the PFC is impractical, but this strategy would rather be approximated with a finite number of anti-solvent addition points. Eq. (5) is expressed in this way to draw an analogy to the more frequently discussed (Nagy et al., 2008; Lindenberg et al., 2009) case of the semi-batch crystallizer for which the PBE can be written as:

$$
\frac{\partial n}{\partial t}=-\frac{\partial(G n)}{\partial L}-\frac{F n}{V}
$$

where $n(t, L)$ is again the number density distribution and $F(t)$ is the volumetric anti-solvent addition rate. Comparing Eqs. (5) and (6) one realizes that for the same anti-solvent addition and temperature profiles (in time for the semi-batch and along the length for the PFC) the same particle size distribution must be obtained from the semi-batch and PFC.

The population balance equations, Eqs. (2), (5) and (6), are coupled with mass balances for the solute. For the $i^{\text {th }}$ MSMPRC at steady state the mass balance can be written as:

$$
0=-3 k_{v} \rho_{c} \int_{0}^{\infty} G L^{2} n \mathrm{~d} L+\frac{Q_{i-1} c_{i-1}-Q_{i} c_{i}}{V_{i}}
$$

where $c_{i}$ is the concentration in the $i^{\text {th }}$ MSMPRC, $\rho_{c}$ is the crystal density and $k_{v}$ is the volumetric shape factor. In the PFC and the semi-batch crystallizer 
the mass balances become:

$$
\begin{aligned}
\frac{Q}{A} \frac{\mathrm{d} c}{\mathrm{~d} z} & =-3 k_{v} \rho_{c} \int_{0}^{\infty} G L^{2} n \mathrm{~d} L-\frac{f c}{A} \\
\frac{\mathrm{d} c}{\mathrm{~d} t} & =-3 k_{v} \rho_{c} \int_{0}^{\infty} G L^{2} n \mathrm{~d} L-\frac{F c}{V}
\end{aligned}
$$

The PBE and mass balance for each process configuration are solved simultaneously. The preferred solution method for these equations depends in general on the form of the constitutive equations describing the nucleation and growth kinetics. For the case studies considered in this paper (see Section 5.1 and Table 1) the method of moments in conjunction with analytical solutions for the steady-state particle size distribution is computationally the most efficient option. A derivation of the solution methods used in this paper is presented in the supplementary material. 


\section{Attainable regions and their construction}

The notion of an attainable region was first introduced by Horn (1964) to the process design of chemical reactors. In such a case, the attainable region consists of all possible outcome states (e.g., a vector of chemical compositions) that results from a given feed stream and chemical reaction scheme (and known kinetics for each of these reactions) when all possible reactor designs are considered, thus accounting for the reaction network, but also for mixing (recycle loops, side streams, mixing of product streams of different reactors, etc.). Thus, conversion and selectivity of different products can be tuned by choosing an appropriate reactor design that lies within this attainable region. This concept has subsequently been investigated extensively and generalized by several researchers (Glasser et al., 1987; Hildebrandt et al., 1990; Hildebrandt and Glasser, 1990; Feinberg and Hildebrandt, 1997; Feinberg, 2002). It has also been applied to problems including reaction, mixing and separation (Nisoli et al., 1997; Feinberg, 2002).

In this article we are interested in the conceptual design of crystallization processes, i.e., we seek to determine the range in which well-defined product characteristics can be altered by changing the crystallizer device and the independent variables describing it. As it was mentioned previously, we will limit ourselves to cascades of MSMPRCs, PFCs and semi-batch crystallizers and will neither investigate combinations of them nor recycle streams. We note that this is not a limitation of the methodology itself, but a choice made for reasons of simplicity. Even in this simplified case, an attainable region for a crystallization process could be calculated in several ways depending on the choice of the dependent state and additional constraints that are considered. Here, we are interested in determining which particle sizes can be attained in a given crystallizer device for given crystallization kinetics and process start and end points. The process start and end points are specified by temperature and antisolvent fraction, i.e., by $\left(T_{0}, a_{0}\right)$ and $\left(T_{f}, a_{f}\right)$, respectively. In order to fix the solute concentration, we specify that the process starts from a saturated solution, i.e., $c_{0}=c_{\star}\left(T_{0}, a_{0}\right)$. In the following, we present a framework that allows constructing the attainable region for an MSMPRC cascade, but at the end of this section we show that this concept can be extended to PFCs and semi-batch crystallizers by introducing minor modifications. To this end, let us start by giving a definition of the mean particle size of the product crystals, $\bar{L}_{m}$ :

$$
\bar{L}_{m}=\frac{\mu_{m, 4}}{\mu_{m, 3}}
$$

In this equation $\mu_{m, j}$ is the $j^{\text {th }}$ moment of the particle size distribution in the $m^{\text {th }}$ (and therefore last) MSMPRC of a cascade, defined as:

$$
\mu_{m, j}=\int_{0}^{\infty} L^{j} n_{m} \mathrm{~d} L
$$


The attainable regions presented in this work will thus specifically answer the question, what range of values can $\bar{L}_{m}$ attain when the independent variables of an MSMPRC cascade are adjusted? The independent variables for the model given in Section 3 are the temperature, the volume fraction of anti-solvent and the residence time (defined as $\tau_{i}=V_{i} / Q_{i}$ ) in each MSMPRC.

In order to obtain economically meaningful processes a yield constraint will be employed, where we will define a thermodynamically achievable yield as:

$$
Y=\frac{Q_{0} c_{0}-Q_{m} c_{m}}{Q_{0} c_{0}-Q_{f} c_{\star, f}}
$$

where $c_{\star, f}\left(T_{f}, a_{f}\right)$ is the solubility at the temperature and anti-solvent fraction at the end point of the crystallization process and $Q_{f}$ is the flow rate that needs to be maintained in order to reach these conditions. It should be noted that this definition of the yield is an alternative to the fraction of recovered solute, $X$ :

$$
X=\frac{Q_{0} c_{0}-Q_{m} c_{m}}{Q_{0} c_{0}}
$$

Enforcing a stringent yield constraint entails that process configurations with the same total residence time in a cascade and the same total crystallizer volume result in production rates between $Y P$ and $P$, where $P$ is a specified production rate (in mass per time). Clearly, the more stringent the yield constraint is chosen, the narrower the interval of obtained production rates. However, the size of the product particles, $\bar{L}_{m}$, resulting from these process configurations might be completely different due to different temperatures and anti-solvent fractions in the crystallizers and a different distribution of the residence time on the crystallizers. Considering the model equations presented in Section 3 one realizes that $\bar{L}_{m}$ depends in a nonlinear way on the independent variables, prohibiting a direct analytical expression for the attainable region. Hence, a convenient way to find the minimal/maximal attainable mean particle size, given a fixed number of MSMPRCs and fixed total residence time in the MSMPRC cascade, is to formulate an optimization problem:

$$
\begin{array}{ll}
\underset{T_{i}, a_{i}, \tau_{i}}{\operatorname{minimize} \text { maximize }} & \bar{L}_{m} \\
\text { subject to } & \sum_{i=1}^{m} \tau_{i}=\tau \\
& T_{i} \leq T_{i-1}, \\
& a_{i} \geq a_{i-1}, \\
& T_{i} \geq T_{f}, \\
& a_{i} \leq a_{f}, \\
& Y \geq 0.98 .
\end{array}
$$

where $i=1, \ldots, m$ and $\tau$ is the total residence time in the MSMPRC cascade 
that is introduced as a constraint. The second and third constraint ensure that the temperature decreases and the anti-solvent fraction increases monotonically along the MSMPRC cascade and the fourth and fifth constraint set the lower bound for the temperature and anti-solvent volume fraction. The last constraint specifies that only process configurations yielding $98 \%$ or more of the thermodynamically achievable yield are valid solutions to the optimization problem. Note that the conditions at the start of the process, i.e., the anti-solvent volume fraction and the temperature in the feed stream, $a_{0}$ and $T_{0}$, are also fixed.

Solving Eq. (14) one obtains the boundary of the attainable region of mean particle sizes vs. total residence time in the MSMPRC cascade. It is an intriguing property of this region that the loci of minimal and maximal mean particle size converge on a single point at low total residence times (see also Section 5.2 and Figure 2). This single point signifies the minimal residence time required to fulfill all the constraints posed in Eq. (14), therefore, this point can be found directly by solving a third optimization problem:

$$
\begin{array}{cl}
\underset{T_{i}, a_{i}, \tau_{i}}{\operatorname{minimize}} & \tau \\
\text { subject to } & \sum_{i=1}^{m} \tau_{i}=\tau \\
& T_{i} \leq T_{i-1}, \\
& a_{i} \geq a_{i-1}, \\
& T_{i} \geq T_{f}, \\
& a_{i} \leq a_{f}, \\
& Y \geq 0.98 .
\end{array}
$$

Note that we cannot report a thorough mathematical proof that the solutions to Eqs. (14) and (15) are unique, however, an intuitive answer to the maximum portion of Eq. (14) can be given straight away: for large total residence times, there exists a unique solution, such that one large particle consists of all crystallized solute mass that equals $100 \%$ of the thermodynamically attainable yield. For shorter total residence times, the crystallization needs to proceed faster, i.e., more nuclei need to be formed in the process, leading to a smaller mean particle size. Clearly, the underlying model equations are nonlinear, but this thought experiment strongly hints at the uniqueness of these solutions. Similar thought experiments can be carried out for the minimum portion of Eq. (14) and for Eq. (15) with the conclusion that the solutions obtained for these optimization problems are also expected to be unique for a given total residence time.

We claim that all intermediate mean particle sizes can be attained by mini- 
mizing the quadratic optimization problem:

$$
\begin{array}{cl}
\underset{T_{i}, a_{i}, \tau_{i}}{\operatorname{minimize}} & \left(\bar{L}_{m, d}-\bar{L}_{m}\right)^{2} \\
\text { subject to } & \sum \tau_{i}=\tau \\
& T_{i} \leq T_{i-1}, \\
& a_{i} \geq a_{i-1}, \\
& T_{i} \geq T_{f}, \\
& a_{i} \leq a_{f} \\
& Y \geq 0.98 .
\end{array}
$$

where $\bar{L}_{m, d}$ is the desired mean particle size. It is again difficult to prove in a mathematically rigorous way that the objective function in the above optimization problem can become zero in all cases. However, we will illustrate this property using several case studies in Section 5. Note that, contrary to Eqs. (14) and (15), Eq. (16) cannot be expected to possess a unique solution as the same mean particle size can be obtained from different particle size distributions. Note that Eqs. (14) to (16) are optimization problems in a $3 m$-dimensional space involving linear and nonlinear constraints. For large values of $m$ (i.e., many crystallizers in the cascade) such problems are expected to exhibit many local minima/maxima. Albeit there is no guarantee to find the global optimum the chances to do so can be significantly increased by using "global optimization" strategies. In our case we have chosen to start a gradient based optimizer (we have used the active-set algorithm built into MATLAB's fmincon (Schittkowski, 1986; MATLAB Optimization toolbox, 2012)) from different starting points (automatically selected by MATLAB's MultiStart (Ugray et al., 2007; MATLAB Global Optimization toolbox, 2012)) so that multiple basins of attraction are analyzed.

To apply the procedure explained above to the construction of attainable regions for PFCs and semi-batch crystallizers one simply redefines $\bar{L}_{m}$ and $Y$ appropriately using properties of the semi-batch crystallizer and the PFC, e.g., the definition of $\bar{L}_{m}$ can be adapted to $\bar{L}\left(t_{\text {batch }}\right)$ and $\bar{L}(Z)$ where the two new quantities represent the mean particle size at the end of a semi-batch process and at the outlet of a PFC, respectively. Clearly, $t_{\text {batch }}$ and $Z$ can be chosen such that the same "total residence time" in the semi-batch crystallizer and the PFC as in the MSMPRC cascade is obtained. Using these definitions for the mean size of the product particles and using the continuous functions $T(t)$ and $a(t)$ or $T(z)$ and $a(z)$ as the independent variables Eqs. (14) and (15) can be reformulated while keeping the constraints analogous to the case of the MSMPRC cascade. In order to simplify the resulting infinite dimensional optimization problems the continuous functions are discretized. The position of the resulting discrete points along the time/length axis, as well as the corresponding temperatures and anti-solvent fractions are then the $3 p$ independent variables of the optimization problem (where $p$ is the number of discrete points). The discrete points are 
connected in a piecewise linear fashion, so that a finite-dimensional optimization problem is obtained that can be solved numerically using the same procedures mentioned above.

\section{Results}

\subsection{Selection of case studies}

To illustrate the methodology presented in Section 4 and discuss the resulting attainable regions, three case studies were selected: the cooling crystallization of paracetamol grown from ethanol, the anti-solvent crystallization of L-asparagine monohydrate from water using isopropanol as the anti-solvent and the combined cooling/anti-solvent crystallization of aspirin from ethanol using water as the anti-solvent. As discussed in Section 4, two key aspects need to be known in order to construct attainable regions: the crystallization kinetics and the process start and end points. The crystallization kinetics (i.e., the constitutive equations describing the growth and nucleation rate) and other model parameters were taken from the literature and are reported in Table 1 (data sources are given as footnotes in the table). These kinetics cover both primary and secondary nucleation mechanisms and different growth rates, so that it can be expected that the attainable regions obtained for these case studies are representative cases for a large number of similar systems. While the start point of a crystallization process is typically the same as the end point of the previous unit operation and is therefore restricted, the choice of the end point is more flexible and is made primarily to maximize the recovered solute fraction, which depends on the phase diagram of the compound in the selected solvent system. However, additional aspects need to be considered as well, e.g., equipment capability (typically limited to $-10^{\circ} \mathrm{C}$ in an industrial setting), maximum allowed suspension density in the crystallizer and the need for impurity rejection. Regarding our case studies, we selected start and end points that we considered to be reasonable, as reported in Table 2. It should be noted that altering this choice would neither change the methodolgy presented above nor substantially change the general conclusions drawn from the results presented below. It would however affect the absolute position of the attainable regions in the residence vs. mean particle size plane.

\subsection{Attainable regions in MSMPRC cascades}

In a first step an exemplary attainable region for a specific MSMPRC cascade will be constructed by solving Eqs. (14) and (15). To this end, we choose an MSMPRC cascade with three crystallizers in which paracetamol is crystallized from ethanol by cooling (as specified in Tables 1 and 2). The solution of Eq. (14) for different total residence times $\tau$ is reported as blue circles in Figure 2. From this figure, one sees that the solutions to the maximum and the minimum part of Eq. (14) both lie on smooth lines that can be traversed by changing the total residence time $\tau$; we will call these two lines the minimum and maximum line in all subsequent descriptions of attainable regions and will depict this boundary of 


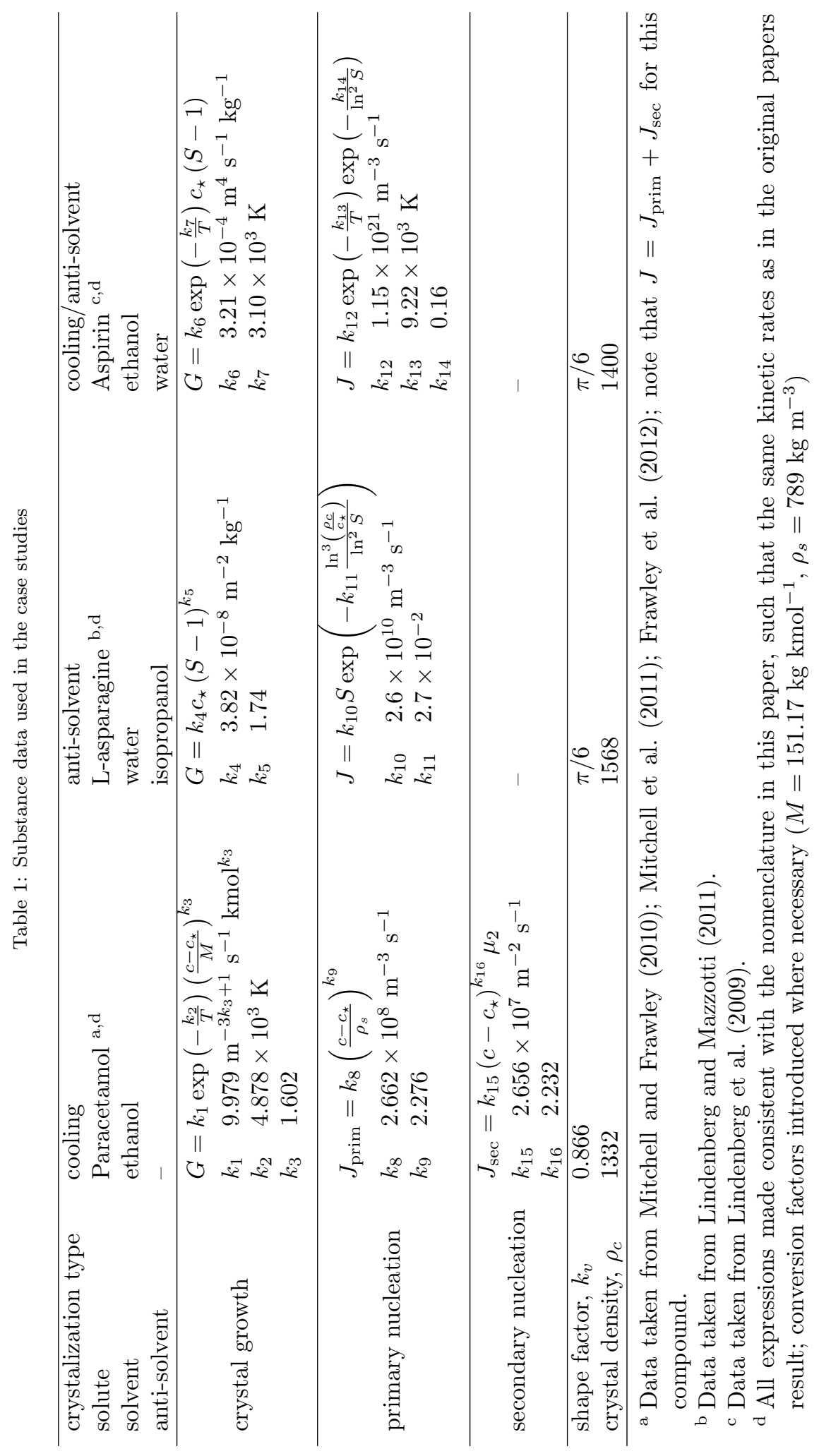


Table 2: Conditions at initial and end points of the case studies

\begin{tabular}{lrrr}
\hline & Paracetamol & L-asparagine & Aspirin \\
start temperature, $T_{0}[\mathrm{~K}]$ & 341 & 298 & 307 \\
end temperature, $T_{f}[\mathrm{~K}]$ & 273 & 298 & 298 \\
start anti-solvent fraction, $a_{0}[-]$ & - & 0 & 0.40 \\
end anti-solvent fraction, $a_{f}[-]$ & - & 0.66 & 0.75 \\
start solubility, $c_{\star}\left(T_{0}, a_{0}\right)\left[\mathrm{kg} \mathrm{m}^{-3}\right]$ & 396 & 29 & 305 \\
end solubility, $c_{\star}\left(T_{f}, a_{f}\right)\left[\mathrm{kg} \mathrm{m}^{-3}\right]$ & 89 & 0.6 & 16 \\
max. solute recovered, $X[-]$ & 0.78 & 0.95 & 0.87 \\
\hline
\end{tabular}

the attainable region as lines rather than a collection of points. The minimum and maximum line converge to a single point for small total residence times, which can be found directly by solving Eq. (15). This point is reported as a red diamond in Figure 2. To the left of this point, i.e., at even smaller total residence times, it is impossible to fulfill the yield constraint implemented in Eqs. (14) and (15) in a cascade of three MSMPRCs for the given crystallization kinetics. One should also appreciate the general behavior of the minimum and maximum line: the minimum attainable particle size stays almost constant when the total residence time is varied. The maximum attainable particle size on the other hand is increasing with total residence time. This increase can be rationalized as the result of added flexibility in the distribution of the total residence time among the three crystallizers while still fulfilling the yield constraint. For very long residence times (not shown in the figure), the physical limit is the formation of one particle consisting of $100 \%$ of the thermodynamically attainable yield.

In Section 4 we claimed that the region between the maximum and minimum line can be fully traversed by changing the values of the independent variables in the MSMPRC cascade. We demonstrate this by solving Eq. (16) for the same cascade of three MSMPRCs discussed above. The attainable region is first traversed horizontally by solving Eq. (16) for $\bar{L}_{m, d}=\{300,400,500,600,700\} \mu \mathrm{m}$ and appropriate values of $\tau$ (such that the points $\left(\bar{L}_{m, d}, \tau\right)$ lie within the previously determined boundaries of the attainable region). The attainable region was then similarly traversed vertically at fixed values of $\tau$ and appropriate values of $\bar{L}_{d, m}$. In all these optimization problems the objective function was reduced to below the convergence limit set for the optimizer (typically $10^{-6} \mu \mathrm{m}^{2}$ ), i.e., the desired particle size was indeed obtained. This can also be seen in Figure 3a by the fact that the intermediate points (open orange circles) lie on straight lines, just as they were intended.

It is now instructive to consider some $\left(\bar{L}_{m, d}, \tau\right)$ combinations in this attainable region and analyze the operating policy that is required to obtain these different mean particle sizes. Three points at a total residence time $\tau=5.6 \mathrm{~h}$ have been highlighted using a red, blue and black marker in Figure 3a and the corresponding operating policies are shown in Figures $3 \mathrm{~b}$ to $3 \mathrm{~d}$. In these operating plots the dashed line is the solubility line, the markers represent the steady state condition of each MSMPRC and the solid line represents the process tra- 


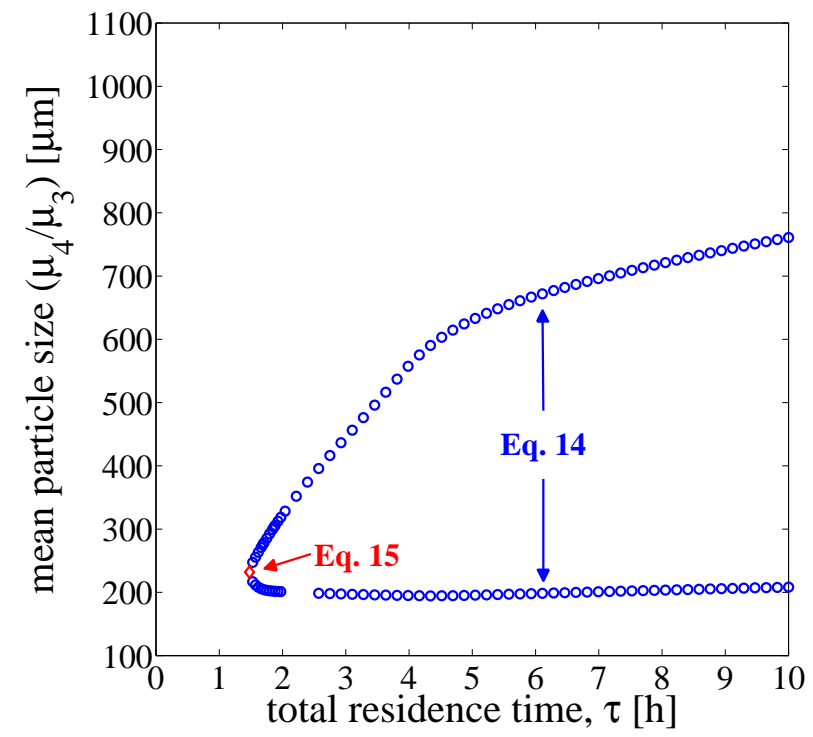

Figure 2: Construction of the attainable region of particle size for a cascade of 3 MSMPRCs in the paracetamol cooling crystallization case study with initial and end point as specified in Table 2. The optimization problems solved to obtain the respective set of points are indicated by arrows.

jectory in this phase diagram. The condition of the feed stream is represented by the point where the solid line originates on the dashed line in each plot (since the start point of the crystallization process is fixed this point is invariable in the three operating plots). The vertical distance between the unmarked corners of the solid lines and the markers for each MSMPRC directly gives the increase in suspension density in each crystallizer. If the residence time in a specific crystallizer were longer, the marker would be located closer to the solubility line, i.e., more solute would crystallize in that crystallizer and a higher suspension density would be observed (the marker would touch the solubility line for an infinitely long residence time). However, note that the underlying (nonlinear) model equations need to be solved in order to determine how much of the solute additionally crystallizes for a certain increase in residence time. Analyzing individual operating plots, one can see that in order to obtain the minimal attainable particle size (Figure $3 \mathrm{~b}$ ) one should operate all three MSMPRCs at the lowest allowed temperature (given by the end point of the process, cf. Table 2), which thus generates the maximum possible supersaturation and therefore induces the highest possible nucleation rate. The distribution of the total residence time among the three crystallizers is not intuitive, but is a result of the interplay of (primary and secondary) nucleation and growth kinetics, which are dependent on the case study considered. The maximal attainable particle size (Figure 3c) is obtained when the first crystallizer is operated at a comparatively high temperature and low supersaturation, so that a smaller 
number of nuclei are generated in the first crystallizer. The remaining two crystallizers are operated such that the remaining yield is consumed mainly by the growth of the initial nuclei while still fulfilling the yield constraint. To arrive at an intermediate mean particle size an operating policy between these two extreme cases needs to be chosen, as shown in Figure 3d.

We have carried out the construction of attainable regions for all three case studies mentioned in Section 5.1 for MSMPRC cascades of two to five crystallizers; the result is shown in Figure 4. Focusing on the paracetamol case study again (Figure 4a) two things are apparent: first, larger attainable regions result when the number of MSMPRCs in the cascade is increased from two to five. This is a consequence of the additional flexiblity provided by more crystallizers. Second, there are diminishing returns for every additional crystallizer added to the cascade, i.e., while the increase in size of the attainable region from two MSMPRCs to three MSMPRCs is substantial, each subsequently added crystallizer yields smaller and smaller gains in the size of the attainable region. The case of an infinite number of (infinitesimally small) MSMPRCs is mathematically equivalent to a PFC and semi-batch crystallizer and is covered in Section 5.3. Considering the attainable regions determined for the remaining two case studies (Figures $4 \mathrm{~b}$ and $4 \mathrm{c}$ ), one sees that these two findings are confirmed. Note that the "kinks" in the maximum line (the points where the line changes slope abruptly), observed prominently in Figure 4b, but also in Figures 4a and 4c, are not coincidental. Focusing for example on the maximum line for a cascade of three MSMPRCs in the L-Asparagine case study (orange maximum line in Figure $4 \mathrm{~b}$ ), this behavior can be understood by considering the actual process configurations obtained by solving the optimization problem. To the left of the kink, fulfilling the constraints in Eq. (14) requires all three crystallizers to be operated at the end point of the crystallization process as the yield constraint could not be fulfilled otherwise. The gain in maximum particle size along this part of the maximum line is the result of the redistribution of the total residence time on the crystallizers. To the right of the kink, the added total residence time allows operating the first crystallizer at a lower anti-solvent fraction while still fulfilling the yield constraint. From this example, one can deduce that (depending on the crystallization kinetics) there might be up to $m-1$ observable kinks in the maximum line for an attainable region of an MSMPRC cascade consisting of $m$ crystallizers.

The three case studies cover a variety of nucleation and growth mechanisms and supersaturation generation methods (cooling, anti-solvent addition and a combination of both), so that one can assume that the above-mentioned findings are independent of the underlying kinetics or the method used to create supersaturation. However, comparing Figures $4 \mathrm{a}$ to $4 \mathrm{c}$, one notices also two pronounced differences between the three case studies: first, the attainable particle sizes, the minimal total residence time and the shape of the attainable regions are significantly different. Second, the gain obtained by adding additional crystallizers to the MSMPRC cascade is also substantially different in each case study. However, the general trend of diminishing returns is still observed. These differences are a consequence of the different crystallization kinetics exhibited 


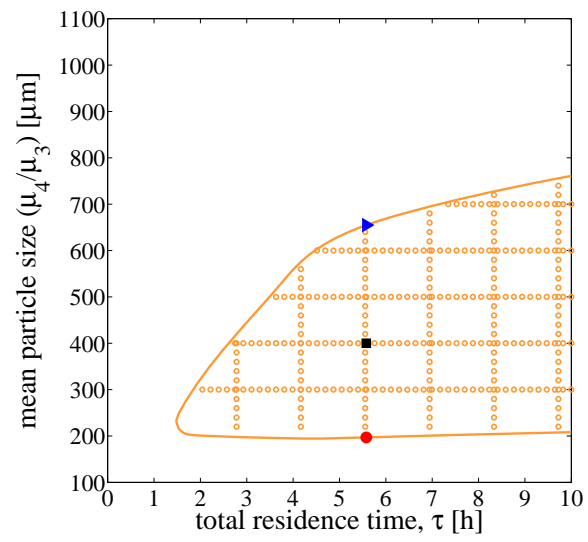

(a)

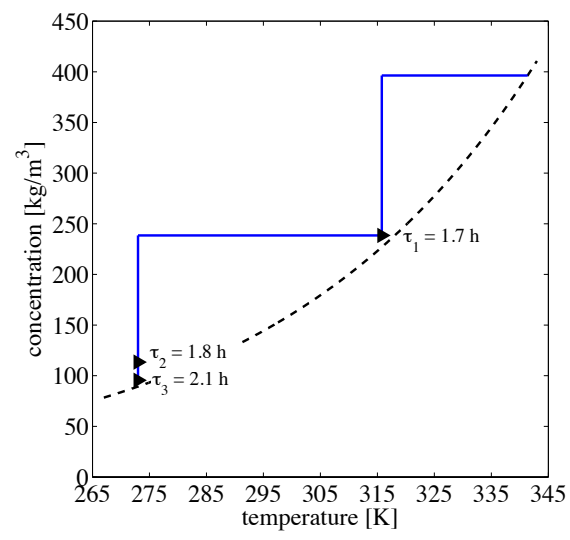

(c)

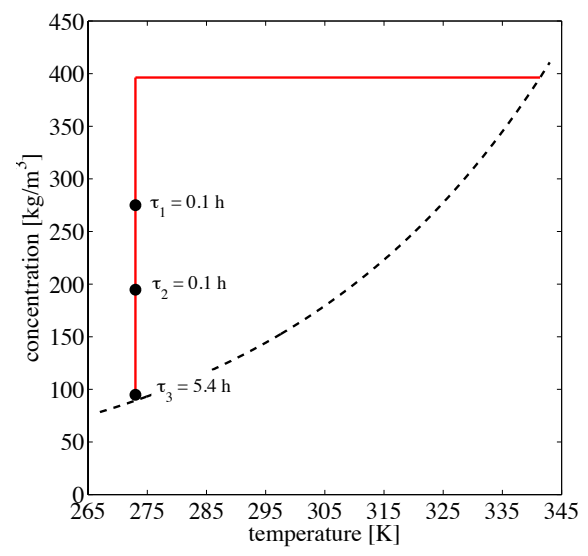

(b)

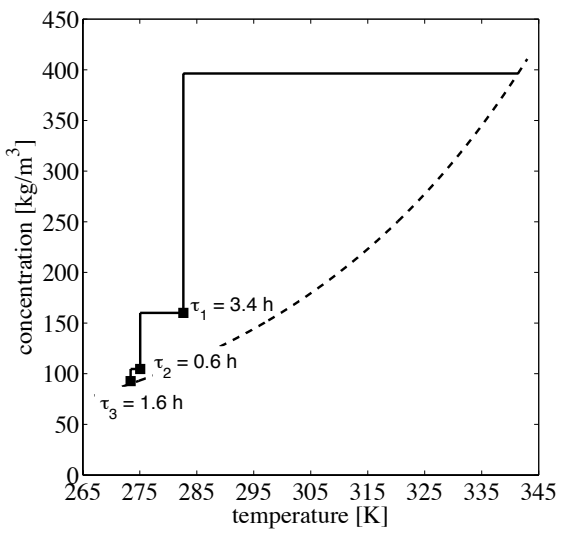

(d)

Figure 3: Attainable region of particle size for a cascade of 3 MSMPRCs in the paracetamol cooling crystallization case study with process start and end point as specified in Table 2: (a) Demonstration that the whole attainable region can be traversed by solving the optimization problem specified in Eq. (16) (open, orange circles). Three specific points at $\tau=5.6 \mathrm{~h}$ are highlighted and their underlying operating policies are shown in the following subfigures; (b) operating policy to obtain the mean particle size marked by the red circle in the attainable region; (c) operating policy to obtain the mean particle size marked by the blue triangle in the attainable region; (d) operating policy to obtain the particle size marked by the black square in the attainable region. In subfigures (b) to (d) the dashed line is the solubility line and markers represent the steady state operating points of each MSMPRC in the cascade. The residence time in each MSMPRC is indicated next to the markers. 
by the three model compounds.

\subsection{Attainable regions in plug flow and semi-batch crystallizers}

In this section attainable regions for PFCs and semi-batch crystallizers are presented for the three case studies and compared to their MSMPRC cascade counterparts. The operational difficulties of a PFC and a semi-batch crystallizer are of a completely different nature and hence require different considerations when an actual process is designed. However, in Section 3 it was shown that they are (as far as the model is concerned) identical when a differential side stream is used in the PFC to describe the anti-solvent addition and when the time and length coordinates are transformed appropriately. Hence, it is sufficient to construct attainable regions only for the PFC, as identical attainable regions would be obtained for a semi-batch crystallizer of equal volume.

In the last paragraph of Section 4 the discretization procedure for the continuous temperature and anti-solvent profiles along the PFC was explained. A large number of discretization points approximates these profiles more accurately, but increases the dimensionality of the optimization problem and thus the necessary computation time to generate an attainable region. In order to analyze this trade off, the operating policy for a PFC leading to the maximal attainable particle size was calculated for different numbers of discretization points (paracetamol case study, $\{5,10,15,20,25,30\}$ discretization points, $\mathrm{PFC}$ residence time of $10 \mathrm{~h}$ ). The resulting operating policies are shown in Figure 5. It is apparent that the operating policies obtained are essentially the same. Moreover, the difference in mean particle size for these operating policies was negligible (less than $2 \%$ variation). It was therefore decided to run all further calculations of attainable regions in the $\mathrm{PFC} /$ semi-batch with 15 discretization points (solid red line in Figure 5).

Having established this, we report the attainable regions for the PFC and semi-batch crystallizer together with their MSMPRC cascade counterparts in Figure 6. Considering first the case of the paracetamol case study (Figure 6a), one can see that the PFC or semi-batch crystallizer allows slightly smaller particles at all residence times and that the maximum attainable particle size increases more steeply at short residence times when compared to the attainable regions of the different MSMPRC cascades. At long residence times however, the MSMPRC cascade consisting of five crystallizers allows obtaining slightly larger maximum particle sizes than the PFC or semi-batch crystallizer. This might be a surprising observation as the attainable regions observed for a low number of MSMPRCs were always larger when more crystallizers were added. However, this is only the case up to a certain number of crystallizers after which the attainable region starts to converge to the semi-batch/PFC attainable region. Considering the model equations reported in Section 3 it is clear that a cascade consisting of an infinite number of MSMPRCs is mathematically equivalent to the PFC and semi-batch crystallizer, i.e., only cascades consisting of an intermediate number of MSMPRCs allow obtaining mean particle sizes larger than in the PFC/semi-batch crystallizer. This convergence behavior was confirmed for the paracetamol case study by solving optimization problems for a large number 


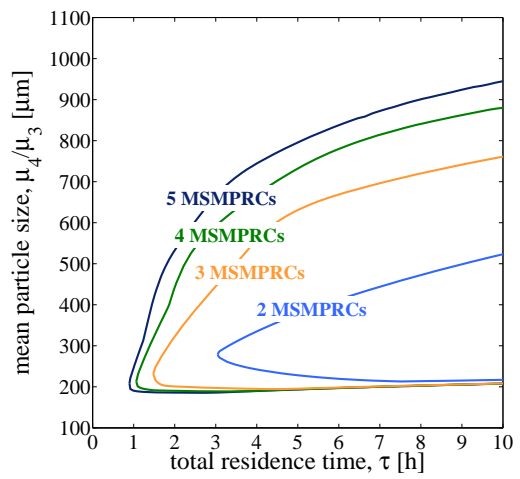

(a)

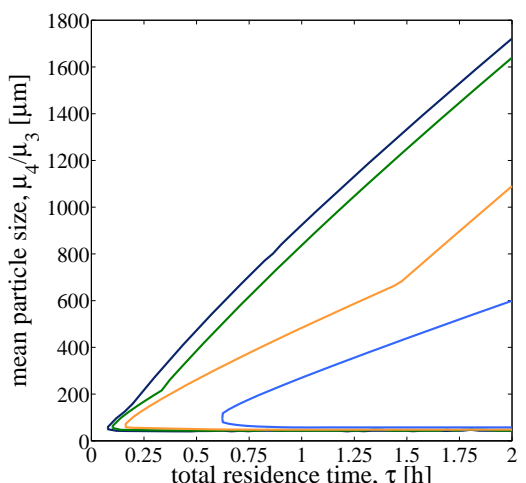

(b)

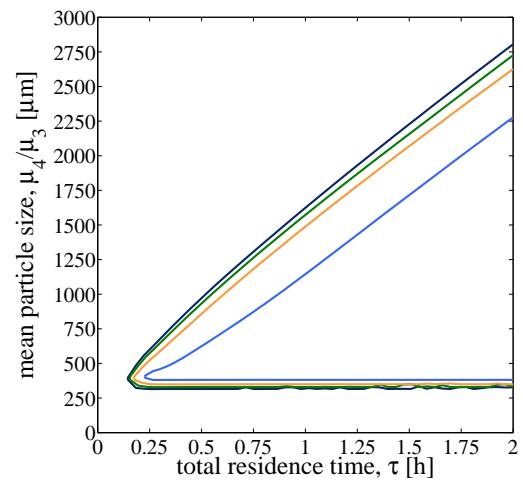

(c)

Figure 4: Attainable particle size regions in MSMPRC cascades consisting of different numbers of MSMPRCs: (a) Paracetamol case study, (b) L-Asparagine monohydrate case study, (c) Aspirin case study. Note that each color is associated with the same number of MSMPRCs in subfigures (a)-(c). The process start and end points of the different case studies are reported in Table 2 . 


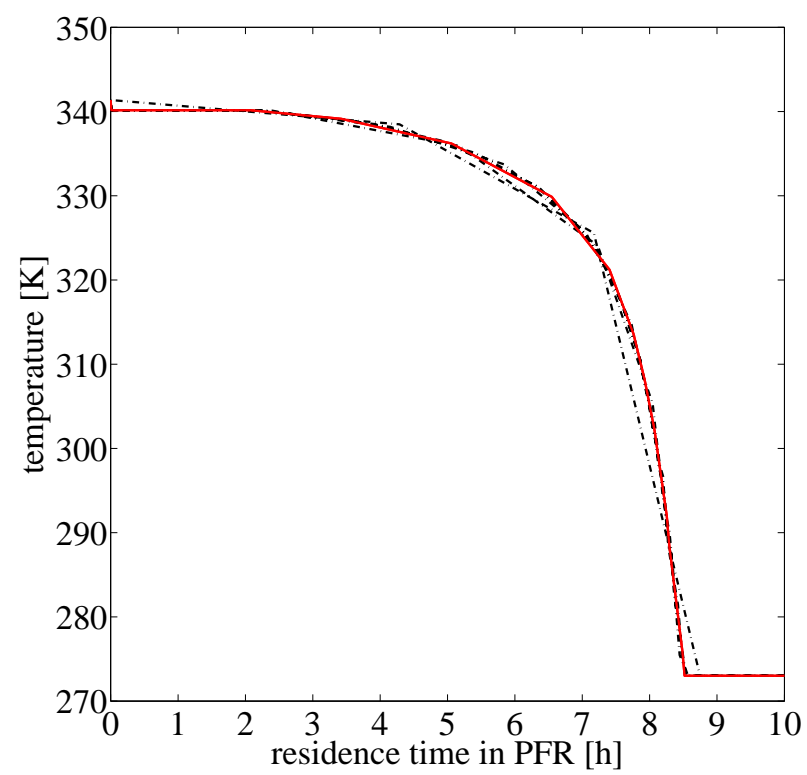

Figure 5: Temperature profile along the PFC in the paracetamol case study for a PFC residence time of $10 \mathrm{~h}$ and process start and end point as reported in Table 2. The different curves represent operating policies obtained for a different number of discretization points $(5$, $10,15,20,25$ and 30 points). The red line represents the case for 15 discretization points that was deemed sufficiently smooth and was thus used in all further calculations. 
of MSMRPCs and it was found that, in this case, the attainable region starts converging to the semi-batch/PFC case between 150 and 200 MSMPRCs. The observations made are reinforced by considering the L-asparagine monohydrate (Figure 6b) and Aspirin (Figure 6c) case studies where this behavior is even more pronounced.

\subsection{Effect of additional operational constraints on the attainable regions}

\subsubsection{Suspension volume constraints}

In the case of continuous crystallization processes scaling down the production rate of a process, while keeping the same product quality, is often an issue. Our attainable regions (cf. Figure 4) indicate that the total residence time in a PFC or MSMPRC cascade can be tuned while still obtaining the same mean size for the product particles, i.e., the attainable region can be traversed horizontally on a line of fixed mean particle size. However, there are caveats associated to this: In a PFC the overall flow rate is limited because at low flow rates the particles will not be properly suspended anymore. Furthermore, traversing the attainable region requires a change in the temperature profile along the length of the PFC, which is difficult to achieve accurately in a real process. In the case of MSMPRC cascades traversing the attainable regions horizontally implies changes in the flow rates and temperatures in each device. The flow rates at which an MSMPRC can be operated are generally more flexible and temperature control is easier as well. However, in the way we have set up our attainable regions, we have put no constraints on the suspension volumes in the crystallizers, so that traversing the attainable region implies that the ratio of suspension volumes in the crystallizers can change in any way along the cascade. For the design of a new process and just for the sake of analyzing the case without additional constraints, such an assumption is reasonable. However, if the production rate needs to be tuned in existing equipment, changes in the suspension volumes are only allowed within certain limits so that the crystallizers are neither running almost empty, nor are getting overfilled, i.e., the crystallizers are usually operated while filled between 20 and $80 \%$ of their total volume. To achieve this, additional constraints can be placed on Eqs. (14) and (15), such as:

$$
\max \left\{\frac{V_{i}}{V_{j}}\right\} \leq \frac{W_{h}}{W_{\ell}}, i, j=1, \ldots, m
$$

where $W_{h}$ and $W_{\ell}$ are the highest and lowest allowed fill fraction in the crystallizers. To investigate the effect of such constraints on the attainable region, the attainable regions for the paracetamol cooling case study with $W_{h} / W_{\ell}=1$, i.e., all crystallizers contain the same suspension volume (and thus have the same residence time in a cooling crystallization), are reported in Figure 7 as solid lines, while the dashed lines represent the unconstrained attainable regions that were reported earlier (the color code is the same as in Figure 4). As one might expect the attainable regions shrink regardless of the number of crystallizers in the cascade by adding this rather stringent constraint. However, while all the attainable regions shrink considerably at small sizes, the maximum lines 


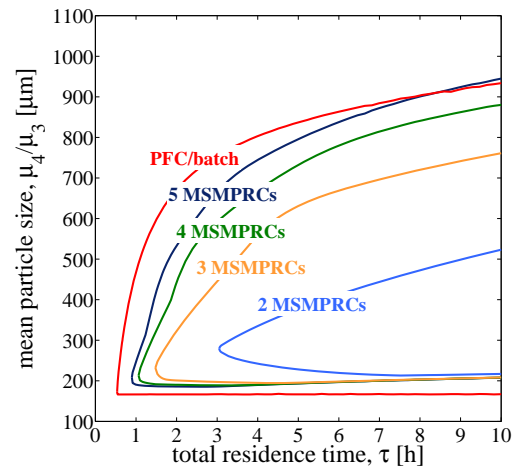

(a)

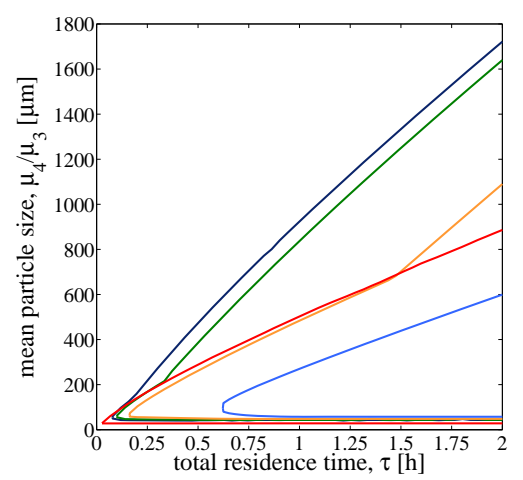

(b)

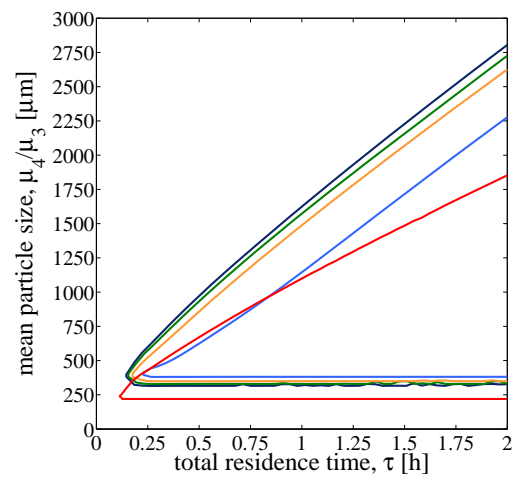

(c)

Figure 6: Attainable particle size regions in PFC/semi-batch crystallizers and MSMPRC cascades consisting of different numbers of MSMPRCs: (a) Paracetamol case study, (b) LAsparagine monohydrate case study, (c) Aspirin case study. Note that red is always associated with the $\mathrm{PFC} /$ semi-batch case and that each other color is associated with the same number of MSMPRCs in subfigures (a)-(c). The process start and points of the different case studies are reported in Table 2. 


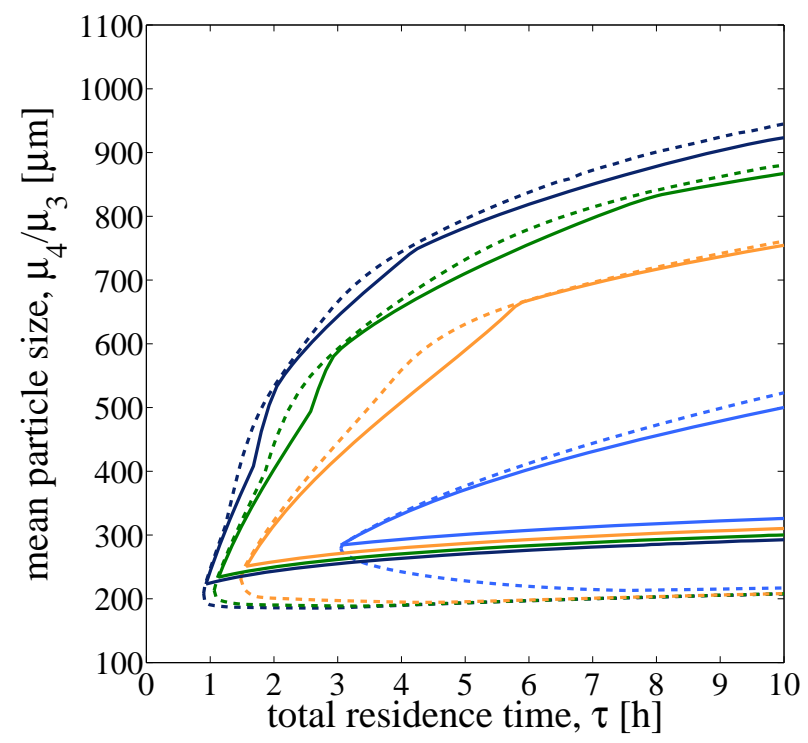

Figure 7: Attainable particle size regions in MSMPRC cascades consisting of different numbers of MSMPRCs for the paracetamol case study when the residence time of each crystallizer can be freely chosen (dashed lines) and when the residence time in each crystallizer in a cascade is the same (solid lines). The color code is the same as in Figure 4.

are barely affected. It should be noted, that the kinks on the maximum line discussed in Section 5.2 occur at lower residence times and are in general more visible. This is reasonable as the constraints placed on process design are more stringent than in Section 5.2.

Since the crystallizers in each MSMPRC cascade now have equal residence time and suspension volume the attainable region can be traversed horizontally, and therefore the production rate can be directly scaled, by simply changing the flow rate (and the temperature in each MSMPRC) while the mean particle size stays the same. In order to show that this is indeed the case the attainable region for a cascade of three MSMPRCs is traversed horizontally as shown in Figure 8a by solving Eq. (16) with Eq. (17) as an additional constraint. One can see that even in the presence of these additional constraints the attainable region can still be traversed.

A word of caution is necessary at this point: The mean particle size is only one characteristic of the complete PSD that could result from, in principle, very different PSDs that result in different product and process characteristics. It therefore makes sense to investigate the full PSDs that result from the different process configurations (each symbol in Figure 8a represents one). It is convenient to use the analytical solution for the steady-state PSD in the third (and 
last) MSMPRC in the above-mentioned cascade: ${ }^{2}$

$$
\begin{aligned}
n_{3}(L) & =\frac{J_{1} G_{1} \tau_{1}^{2}\left(\exp \left(-\frac{L}{G_{1} \tau_{1}}\right)-\exp \left(-\frac{L}{G_{3} \tau_{3}}\right)\right)}{\left(G_{1} \tau_{1}-G_{2} \tau_{2}\right)\left(G_{1} \tau_{1}-G_{3} \tau_{3}\right)}+ \\
& +\frac{J_{1} \tau_{1} G_{2} \tau_{2}\left(\exp \left(-\frac{L}{G_{3} \tau_{3}}\right)-\exp \left(-\frac{L}{G_{2} \tau_{2}}\right)\right)}{\left(G_{1} \tau_{1}-G_{2} \tau_{2}\right)\left(G_{1} \tau_{1}-G_{3} \tau_{3}\right)}+ \\
& +\frac{J_{2} \tau_{2}\left(\exp \left(-\frac{L}{G_{2} \tau_{2}}\right)-\exp \left(-\frac{L}{G_{3} \tau_{3}}\right)\right)}{G_{2} \tau_{2}-G_{3} \tau_{3}}+ \\
& +\frac{J_{3}}{G_{3}} \exp \left(-\frac{L}{G_{3} \tau_{3}}\right)
\end{aligned}
$$

where $G_{i}$ and $J_{i}$ are the growth and nucleation rate that result from the process conditions in the $i^{\text {th }}$ MSMPRC (see supplementary information for details). The PSDs resulting from every process configuration in Figure 8a are shown in Figure 8b. One can see that for process configurations that yield the same mean particle size (solid lines with the same color in Figure 8b) the particle size distributions are only marginally different. This means that the production rate in such a continuous crystallization process can be tuned over a wide range without changing the product particle size distribution simply by changing the flow rate through the cascade and the temperature in the crystallizers (which are found by solving the optimization problem mentioned above).

\subsubsection{Maximum supersaturation constraints}

Another constraint that could be introduced to the attainable regions are constraints on the maximum allowed supersaturation. The underlying reasons for introducing such constraints are twofold: First, a high supersaturation often leads to the formation of strongly agglomerated particles which can have disadvantageous processing properties or purity issues (Hounslow et al., 2001). Second, some organic compounds are known to exhibit metastable liquid/liquid phase separations (LLPS; colloquially also referred to as oiling out) at high supersaturations (Bonnett et al., 2003; Lafferrere et al., 2004; Deneau and Steele, 2005; Codan et al., 2010), which is also a potential route to crystals of low purity and quality. Considering these effects, it makes sense to limit the maximum allowed supersaturation using a constraint. For an MSMPRC cascade we therefore write:

$$
S_{i} \leq S_{\text {thres }}, \quad \text { for } \quad i=1, \ldots, m
$$

where $S_{i}$ is the supersaturation in the $i^{\text {th }}$ MSMPRC and $S_{\text {thres }}$ is a compound specific threshold supersaturation. As an example, an MSMPRC cascade consisting of three MSMPRCs for the paracetamol cooling crystallization case study

\footnotetext{
${ }^{2}$ The solution reported here is different from the one reported in Alvarez et al. (2011). The detailed derivation is given in the supplementary material.
} 


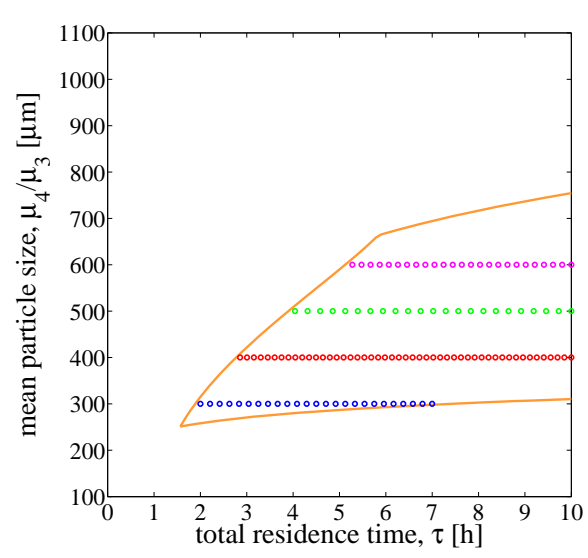

(a)

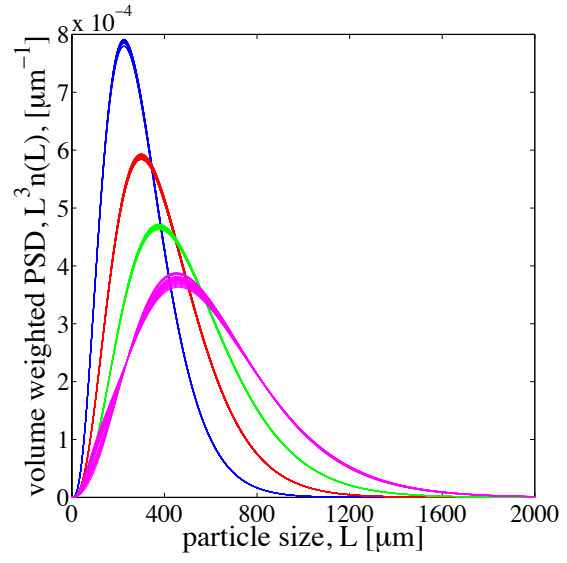

(b)

Figure 8: Attainable regions for an MSMPRC cascade of three crystallizers containing equal suspension volumes for the paracetamol cooling crystallization case study: a) attainable region (solid, orange lines) with intermediate points (blue, red, green and magenta circles), b) PSDs leaving the third MSMPRC for the intermediate points (colors of the PSDs correspond to the colors of the intermediate points in subfigure a).

will be considered again. The attainable regions for this MSMPRC cascade were calculated for different threshold supersaturations $\left(S_{\text {thres }}=\{1.5,2\}\right)$; they are reported in Figure 9 together with the attainable region without additional supersaturation constraint. One sees that the supersaturation threshold progressively moves the minimum line upwards, i.e., the exclusion of high supersaturations removes the ability to form the smallest particle sizes (that are possible with the given kinetics). This result is fully consistent with the fact that the operating policy identified to yield the smallest particles employs the highest possible supersaturations in the cascade (c.f. Figure $3 \mathrm{~b}$ and discussion in Section 5.2). On the other hand, the introduction of a threshold supersaturation does not affect the maximum line of the attainable region at all.

\subsubsection{Yield constraints}

For all the attainable regions presented so far a yield of $Y \geq 0.98$ has been enforced. In order to investigate the effect of different values for the yield constraint attainable regions for an MSMPRC cascade consisting of three crystallizers for the paracetamol cooling crystallization case study with yield constraints of 0.94 and 0.90 are reported in Figure 10. One sees that relaxing the yield constraint allows more flexibility in the production of large particles. This is the result of a trade off between nucleation and growth kinetics: at a given total residence time the deposition of more mass on the crystals would lead to larger crystals if the number of crystals stays constant, but in order to fulfill the yield constraint the crystallizers are operated at a higher supersaturation which results in more crystals that are, on average, of smaller size than their 


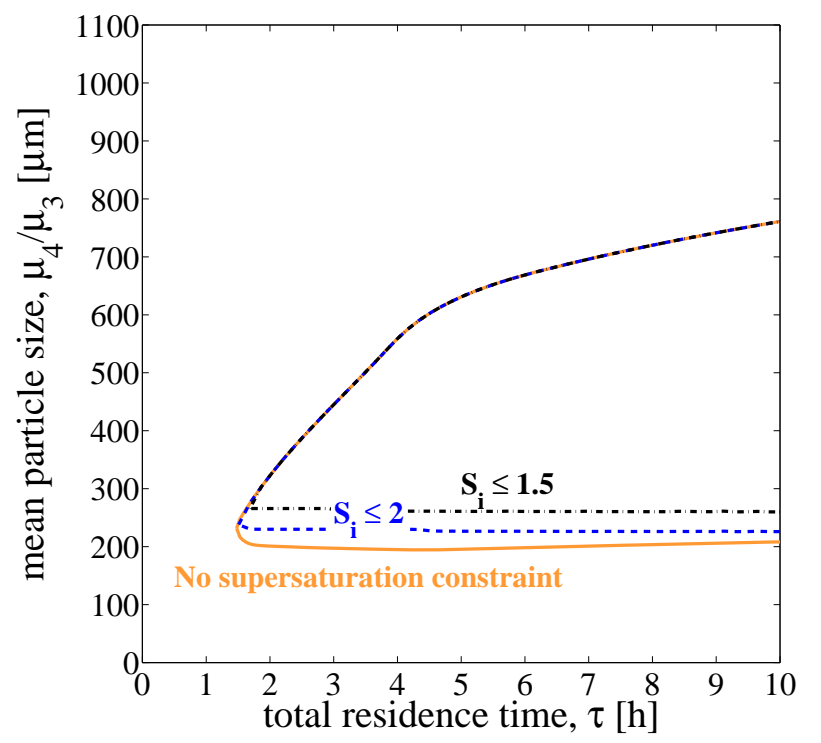

Figure 9: Effect of maximum supersaturation constraint on the attainable region for an MSMPRC cascade consisting of three crystallizers for the paracetamol cooling crystallization case study.

counterparts produced when a lower yield constraint is enforced. 


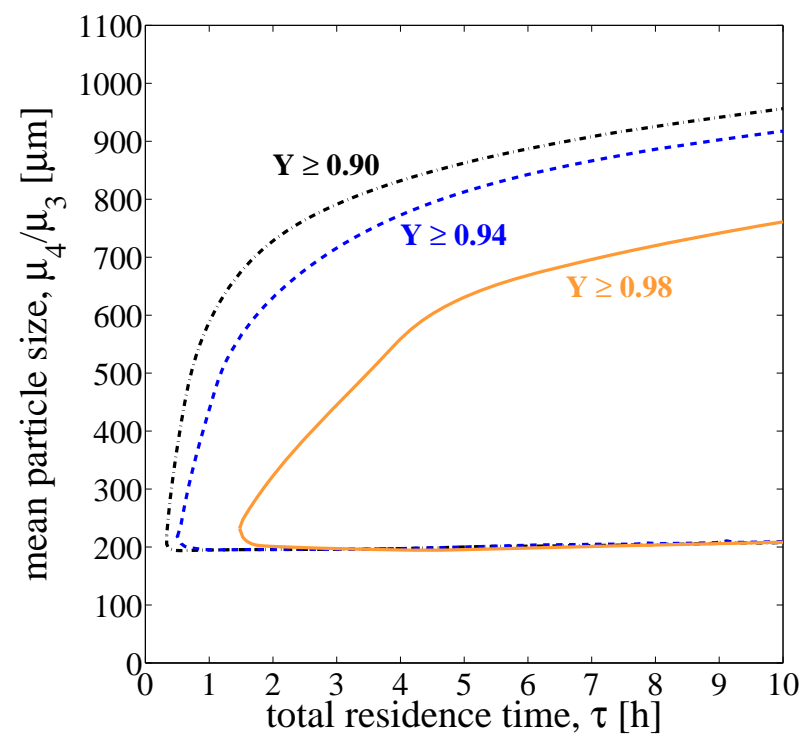

Figure 10: Effect of yield constraint on the attainable region for an MSMPRC cascade of three crystallizers for the paracetamol cooling crystallization case study. 


\section{Concluding Remarks}

This paper presents a methodology that allows finding regions of attainable particle sizes in crystallization processes. All that is required to apply this methodology are crystallization kinetics and defined process start and end points. We specifically reported such regions for MSMPRC cascades, plug flow and semi-batch crystallizers, where the former two were operated without any recycling operations, for case studies where supersaturation is generated using cooling, anti-solvent addition or combinations of the two techniques to induce crystallization. In these case studies we found that adding more crystallizers to an MSMPRC cascade first increases the size of the attainable region before it starts to shrink to the attainable region for the $\mathrm{PFC} /$ semi-batch attainable region for a large number of MSMPRCs. MSMPRC cascades consisting of a low number of crystallizers allows obtaining significantly larger product particle sizes (over a large range of total residence times) compared to the plug flow and semi-batch crystallizers. We note that the methodology to find attainable regions could also be applied to combinations of the above-mentioned crystallizer types, for flowsheets including recycle operations, and virtually any other process equipment or supersaturation generation method as long as the crystallization kinetics for the substance considered are available and the process equipment can be modeled.

The methodology is also flexible enough to incorporate other types of constraints on the process. We specifically investigated constraints on the ratio of volumes of the crystallizers (in order to prevent under- and overfilling of the crystallizers) and reported attainable regions for MSMPRC cascades of equalsized crystallizers. In these attainable regions the production rate becomes fully scalable (within bounds) without affecting the product quality. Since the attainable regions found for the different compounds investigated in the case studies show considerable overlap, this finding hints at the possibility to run continuous crystallization processes for different compounds in the same MSMPRC cascade simply by tuning the independent variables in an appropriate way. We also investigated the influence of yield constraints and constraining the maximum allowed supersaturation on the attainable regions and found that they mainly influence the maximum and minimum lines of the attainable regions, respectively.

Knowing the attainable region allows the prudent engineer to assess if desired particle size specifications can be met in existing process equipment or, if a new plant is to be designed, what process equipment is needed to meet these specifications. 


\section{Acknowledgements}

Research was supported in part by a Lilly Innovation Fellowship Award to TV from Eli Lilly and Company. 


\section{Notation}

\begin{tabular}{|c|c|c|}
\hline$A$ & $\begin{array}{l}\text { volume fraction (solute free basis) of anti-solvent } \\
\text { cross sectional area of PFC }\end{array}$ & $\begin{array}{l}{[-]} \\
{\left[\mathrm{m}^{2}\right]}\end{array}$ \\
\hline$B$ & birth terms in the PBE & {$\left[\mathrm{m}^{-4} \mathrm{~s}^{-1}\right]$} \\
\hline$c$ & solute concentration (solute free basis) & {$\left[\mathrm{kg} \mathrm{m}^{-3}\right]$} \\
\hline$c_{\star}$ & solubility & {$\left[\mathrm{kg} \mathrm{m}^{-3}\right.$} \\
\hline$\hat{D}$ & death terms in the PBE & {$\left[\mathrm{m}^{-4} \mathrm{~s}^{-1}\right]$} \\
\hline$F_{i}$ & volumetric flow rate of anti-solvent stream to $i^{\text {th }}$ MSMPRC & {$\left[\mathrm{m}^{3} \mathrm{~s}^{-1}\right]$} \\
\hline$F(t)$ & $\begin{array}{l}\text { volumetric flow rate of anti-solvent stream to semi-batch } \\
\text { crystallizer }\end{array}$ & {$\left[\mathrm{m}^{3} \mathrm{~s}^{-1}\right]$} \\
\hline$f(z)$ & differential anti-solvent side stream to PFC & {$\left[\mathrm{m}^{2} \mathrm{~s}^{-1}\right]$} \\
\hline$G$ & crystal growth rate & {$\left[\mathrm{m} \mathrm{s}^{-1}\right]$} \\
\hline$J$ & nucleation rate & {$\left[\mathrm{m}^{-3} \mathrm{~s}^{-1}\right]$} \\
\hline$k_{i}$ & parameter in kinetic expression, c.f. Table 1 & [varies] \\
\hline$k_{v}$ & volumetric shape factor & {$[-]$} \\
\hline$L$ & crystal size & [m] \\
\hline $\bar{L}$ & mean crystal size, $\mu_{4} / \mu_{3}$ & [m] \\
\hline$m$ & number of MSMPRCs in the cascade & [-] \\
\hline$m_{f}$ & mass of isolated crystalline product & {$[\mathrm{kg}]$} \\
\hline$m_{f}$ & mass of seed crystals & {$[\mathrm{kg}]$} \\
\hline$M$ & molar mass & [-] \\
\hline$n$ & number density distribution & {$\left[\mathrm{m}^{-4}\right]$} \\
\hline$p$ & $\begin{array}{l}\text { number of discretization points for a } \mathrm{PFC} / \text { semi-batch pro- } \\
\text { file }\end{array}$ & {$[-]$} \\
\hline$Q$ & volumetric flow rate & {$\left[\mathrm{m}^{3} \mathrm{~s}^{-1}\right]$} \\
\hline$t$ & time & [s] \\
\hline$S$ & supersaturation, $c / c_{\star}$ & {$[-]$} \\
\hline$T$ & temperature & {$[\mathrm{K}]$} \\
\hline$V$ & volume of suspension & {$\left[\mathrm{m}^{3}\right]$} \\
\hline$W$ & fill fraction of a crystallizer & [-] \\
\hline$X$ & fraction of recovered solute & [-] \\
\hline$Y$ & fraction of attainable yield & [-] \\
\hline$z$ & length coordinate along PFR & [m] \\
\hline$Z$ & length of PFC & [m] \\
\hline$\tau$ & $\begin{array}{l}\text { total residence time in MSMPRC cascade, PFC or semi- } \\
\text { batch crystallizer }\end{array}$ & {$[\mathrm{s}]$} \\
\hline$\tau_{i}$ & residence time in $i^{\text {th }}$ MSMPRC, $V_{i} / Q_{i}$ & {$[\mathrm{~s}]$} \\
\hline c & crystal density & {$\left[\mathrm{kg} \mathrm{m}^{-3}\right]$} \\
\hline$s$ & suspension density & {$\left[\mathrm{kg} \mathrm{m}^{-3}\right]$} \\
\hline$u_{j}$ & $j^{\text {th }}$ moment of number density distribution & {$\left[\mathrm{kg} \mathrm{m}^{j-3}\right]$} \\
\hline
\end{tabular}




\section{References}

Alvarez, A.J., Myerson, A.S., 2010. Continuous Plug Flow Crystallization of Pharmaceutical Compounds. Cryst. Growth Des. 10, 2219-2228.

Alvarez, A.J., Singh, A., Myerson, A.S., 2011. Crystallization of Cyclosporine in a Multistage Continuous MSMPR Crystallizer. Cryst. Growth Des. 11, $4392-4400$.

Bonnett, P., Carpenter, K., Dawson, S., Davey, R., 2003. Solution crystallisation via a submerged liquid-liquid phase boundary: oiling out. Chem. Commun. , 698-699.

Brazeau, G., Sauberan, S.L., Gatlin, L., Wisniecki, P., Shah, J., 2011. Effect of particle size of parenteral suspensions on in vitro muscle damage. Pharm. Dev. Technol. 16, 591-598.

Cavalcante, P., Dondi, M., Guarini, G., Raimondo, M., Baldi, G., 2009. Colour performance of ceramic nano-pigments. Dyes Pigments 80, 226-232.

Chen, J., Sarma, B., Evans, J.M., Myerson, A.S., 2011. Pharmaceutical Crystallization. Cryst. Growth Des. 11, 887-895.

Chung, S.H., Ma, D.L., Braatz, R.D., 1999. Optimal Seeding in Batch Crystallization. Can. J. Chem. Eng. 77, 590-596.

Codan, L., Bäbler, M.U., Mazzotti, M., 2010. Phase Diagram of a Chiral Substance Exhibiting Oiling Out in Cyclohexane. Cryst. Growth Des. 10, 40054013.

Deneau, E., Steele, G., 2005. An In-Line Study of Oiling Out and Crystallization. Org. Process Res. Dev. 9, 943-950.

Eder, R.J., Radl, S., Schmitt, E., Innerhofer, S., Maier, M., Gruber-Woelfler, H., Khinast, J.G., 2010. Continuously Seeded, Continuously Operated Tubular Crystallizer for the Production of Active Pharmaceutical Ingredients. Cryst. Growth Des. 10, 2247-2257.

Feinberg, M., 2002. A Geometric Approach to Steady Flow Reactors: The Attainable Region and Optimization in Concentration Space. Ind. Eng. Chem. Res. 41, 3751-3761.

Feinberg, M., Hildebrandt, D., 1997. Optimal reactor design from a geometric viewpoint I. Universal properties of the attainable region. Chem. Eng. Sci. $62,1637-1665$.

Frawley, P.J., Mitchell, N.A., Ó'Ciardhá, C.T., Hutton, K.W., 2012. The effects of supersaturation, temperature, agitation and seed surface area on the secondary nucleation of paracetamol in ethanol solutions. Chem. Eng. Sci. 78, 183-197. 
Genck, W., 2003. Optimizing Crystallizer Scaleup. Chem. Eng. Prog. 99, 36-44.

Glasser, D., Hildebrandt, D., Crowe, C., 1987. A Geometric Approach to Steady Flow Reactors: The Attainable Region and Optimization in Concentration Space. Ind. Eng. Chem. Res. 26, 1803-1810.

Griffin, D.W., Mellichamp, D.A., Doherty, M.F., 2010. Reducing the mean size of API crystals by continuous manufacturing with product classification and recycle. Chem. Eng. Sci. 65, 5770-5780.

Hildebrandt, D., Glasser, D., 1990. The attainable region and optimal reactor structures. Chem. Eng. Sci. 45, 2162-2168.

Hildebrandt, D., Glasser, D., Crowe, C., 1990. Geometry of the Attainable Region Generated by Reaction and Mixing: With and without Constraints. Ind. Eng. Chem. Res. 29, 49-58.

Horn, F., 1964. Attainable and Non-Attainable Regions in Chemical Reaction Technique. In Proceedings of the Third European Symposium on Chemical Reaction Engineering, 293.

Hounslow, M., Mumtaz, H., Collier, A., Barrick, J., Bramley, A., 2001. A micro-mechanical model for the rate of aggregation during precipitation from solution. Chem. Eng. Sci. 56, 2543-2552.

Kadam, S.S., Kramer, H.J., ter Horst, J.H., 2011. Combination of a Single Primary Nucleation Event and Secondary Nucleation in Crystallization Processes. Cryst. Growth Des. 11, 1271-1277.

Kadam, S.S., Kulkarni, S.A., Ribera, R.C., Stankiewicz, A.I., ter Horst, J.H., Kramer, H.J., 2012. A new view on the metastable zone width during cooling crystallization. Chem. Eng. Sci 12, 3036-3044.

Kougoulos, E., Smales, I., Verrier, H.M., 2011. Towards Integrated Drug Substance and Drug Product Design for an Active Pharmaceutical Ingredient Using Particle Engineering. AAPS PharmSciTech 12, 287-294.

Lafferrere, L., Hoff, C., Veesler, S., 2004. In Situ Monitoring of the Impact of Liquid-Liquid Phase Separation on Drug Crystallization by Seeding. Cryst. Growth Des. 4, 1175-1180.

Lakatos, B.G., 1996. Uniqueness and multiplicity in isothermal CMSMPR crystallizers. AIChE J. 42, 285-289.

Lakatos, B.G., Sapundzhiev, T.J., Garside, J., 2007. Stability and dynamics of isothermal CMSMPR crystallizers. Chem. Eng. Sci. 62, 4348-4364.

Larsen, P.A., Patience, D.B., Rawlings, J.B., 2006. Industrial crystallization process control. IEEE Control Syst. Mag 26, 70-80. 
Lawton, S., Steele, G., Shering, P., Zhao, L., Laird, I., Ni, X.W., 2009. Continuous Crystallization of Pharmaceuticals Using a Continuous Oscillatory Baffled Crystallizer. Org. Process Res. Dev. 13, 1357-1363.

Lindenberg, C., Krättli, M., Cornel, J., Mazzotti, M., Brozio, J., 2009. Design and Optimization of a Combined Cooling/Antisolvent Crystallization Process. Cryst. Growth Des. 9, 1124-1136.

Lindenberg, C., Mazzotti, M., 2011. Continuous Precipitation of L-Asparagine Monohydrate in a Micromixer: Estimation of Nucleation and Growth Kinetics. AIChE J. 57, 942-950.

Liu, Y., Yin, H., Yuan, S., Chen, Z., 2010. Influence of particle characteristics and $\mathrm{E} / \mathrm{Z}$-isomer ratio on the colour of concentrated $\beta$-carotene dispersions. Int. J. Food. Sci. Tech. 45, 1450-1456.

MATLAB Global Optimization toolbox, 2012. version 3.2.2 (R2012b). The MathWorks Inc., Natick, Massachusetts.

MATLAB Optimization toolbox, 2012. version 6.2.1 (R2012b). The MathWorks Inc., Natick, Massachusetts.

Mersmann, A., 2001. Crystallization Technology Handbook. Marcel Dekker, New York.

Mitchell, N.A., Frawley, P.J., 2010. Nucleation kinetics of paracetamol-ethanol solutions from metastable zone widths. J. Cryst. Growth 312, 2740-2746.

Mitchell, N.A., Ó'Ciardhá, C.T., Frawley, P.J., 2011. Estimation of the growth kinetics for the cooling crystallisation of paracetamol and ethanol solutions. J. Cryst. Growth 328, 39-49.

Nagy, Z.K., Braatz, R.D., 2012. Advances and New Directions in Crystallization Control. Annu. Rev. Chem. Biomol. Eng. 3, 55-75.

Nagy, Z.K., Chew, J.W., Fujiwara, M., Braatz, R.D., 2008. Comparative performance of concentration and temperature controlled batch crystallizations. J. Process Contr. 18, 399-407.

Nisoli, A., Malone, M.F., Doherty, M.F., 1997. Attainable Regions for Reaction with Separation. AIChE J. 43, 374-387.

Quon, J.L., Zhang, H., Alvarez, A., Evans, J., Myerson, A.S., Trout, B.L., 2012. Continuous Crystallization of Aliskiren Hemifumarate. Cryst. Growth Des. $12,3036-3044$.

Ramkrishna, D., 2000. Population Balances: Theory and Applications to Particulate Systems in Engineering. Academic Press, San Diego. 
Randolph, A.D., Larson, M.A., 1988. Theory of Particulate Process: Analysis and Techniques of Continuous Crystallization. 2nd ed., Academic Press, New York.

Rawlings, J.B., Miller, S.M., Witkowski, W.R., 1993. Model Identification and Control of Solution Crystallization Processes: A Review. Ind. Eng. Chem. Res. 32, 1275-1296.

Schittkowski, K., 1986. NLPQL: a Fortran subroutine solving constrained nonlinear programming problems. Ann. Oper. Res. 5, 485-500.

Sherwin, M., Shinnar, R., Katz, S., 1967. Dynamic Behavior of the Well-Mixed Isothermal Crystallizer. AIChE J. 13, 1141-1154.

Ugray, Z., Lasdon, L., Plummer, J.C., Glover, F., Kelly, J., Martì, R., 2007. Scatter Search and Local NLP Solvers: A Multistart Framework for Global Optimization. INFORMS J. Comput. 19, 328-340.

Ward, J.D., Cheng-Ching, Y., Doherty, M.F., 2011. A New Framework and a Simpler Method for the Development of Batch Crystallization Recipes. AIChE J. 57, 606-617.

Ward, J.D., Mellichamp, D.A., Doherty, M.F., 2006. Choosing an operating policy for seeded batch crystallization. AIChE J. 52, 2046-2054.

Wong, S.Y., Tatusko, A.P., Trout, B.L., Myerson, A.S., 2012. Development of Continuous Crystallization Processes Using a Single-Stage Mixed-Suspension, Mixed-Product Removal Crystallizer with Recycle. Cryst. Growth Des. 12, 5701-5707.

Woo, X.Y., Tan, R.B., Braatz, R.D., 2011. Precise tailoring of the crystal size distribution by controlled growth and continuous seeding from impinging jet crystallizers. CrystEngComm 13, 2006-2014.

Yu, K., Douglas, J., 1975. Self-Generated Oscillations in Continuous Crystallizers: Part 1. Analytical Prediction of the Oscillating Output. AIChE J. 21, 917-924.

Zhang, H., Quon, J., Alvarez, A.J., Evans, J., Myerson, A.S., Trout, B.L., 2012. Development of Continuous Anti-Solvent/Cooling Crystallization Process using Cascaded Mixed Suspension, Mixed Product Removal Crystallizers. Org. Process Res. Dev. 16, 915-924. 